daß die Glucose anscheinend der spezifische Reiz für die Insulinabgabe ist, muß man schließen, daß die Erhöhung der Blutglucose direkt oder wahrscheinlicher indirekt die Ursache für die Änderung der Zinkbindung des Insulins ist. Nach $\mathrm{He} f \mathrm{fte} r$ hat Zink eine spezifisch chemische Affinität zu verschiedenen biologischen Schwermetallkomplexbildnern, zu Aminosäuren, Zuckerphosphatsäuren, Lipoiden, Brenzcatechinen, deren Derivaten, SH-haltigen Substanzen und zu Derivaten des Blutfarbstoffs ${ }^{23}$. Ähnliche Substanzen kommen als konkurrierende Komplexbildner mit dem Insulin-Zink in Frage. Vielleicht deutet die Beobachtung von $\mathrm{S}$ ti e r auf die intrainsuläre Anreicherung derartiger Substanzen; er fand, daß die Langerhansschen Inseln von normalen Tieren Triphenyltetrazoliumchlorid kaum reduzieren, während die Inseln mit Glucose belasteter Tiere eine wesentlich stärkere Formazanbildung zeigen ${ }^{24}$.

In gewisser Weise analog zu den Beobachtungen bei der Entstehung des Dithizon- und Alloxandiabetes lassen sich die Vorgänge bei der basalen Insulinregulation durch die Blutglucose bisher wie folgt formu-

23 Hdbch. exp. Pharmakol., 3. Bd., 3. Tl., S. 1909 [1934].

24 A. S ti e r, Z. Anatomie Entwicklungsgesch. 116, 399 [1952]. lieren: Das Insulin liegt auf Grund seiner Zinkbindung in unlöslicher Form in den Inseln vor. Nach der Erhöhung des Blutglucosespiegels kommt es an der Oberfläche der Granula zu einer konkurrierenden Komplexbildung zwischen dem Insulinzink einerseits und Substanzen, die infolge der Vermehrung der Blutglucose auftreten, andererseits. Dadurch wird das Insulin in eine lösliche Form übergeführt und somit die Voraussetzung für dessen Abgabe an das Blut gegeben.

Bei der ausschließlichen Betrachtung der sogenannten basalen Insulinregulation wurden in der vorliegenden Arbeit bewußt weitere Faktoren außer acht gelassen, die im Gesamtorganismus sicher diese Vorgänge zusätzlich beeinflussen, wie z. B. zentral-nervöse und weitere hormonale Einflüsse.

Diese Untersuchungen erscheinen auch von Interesse für die Klinik des Diabetes, da Störungen der Insulinregulation leicht zu einem Mangel an Insulinreserven oder direkt zu dem Bild eines Insulinmangelzustandes führen können.

Die Untersuchungen wurden durch die Hilfe der Deutschen Forschungsgemeinschaft ermöglicht.

\title{
NOTIZEN
}

Zur Kenntnis von Siliciumditellurid (II. Mitt. über Siliciumchalkogenide) ${ }^{1}$

Von Alarich Weis und Armin Weis s

Eduard-Zintl-Institut für anorganische und physikalische Chemie der Technischen Hochschule Darmstadt

(Z. Naturforschg. 8 b, 104 [1953]; eingeg. am 26. Januar 1953)

Siliciumditellurid, $\mathrm{SiTe}_{2}$, wurde durch Erhitzen von Silicium und Tellur auf $1050-1070^{\circ} \mathrm{C}$ im Hochvakuum dargestellt. Die Verbindung bildet rote Plättchen, die sich an der Luft unter Bildung von $\mathrm{SiO}_{2}$ und $\mathrm{H}_{2}$ Te zersetzen. Bei der Zersetzung im Hochvakuum bei etwa $1200^{\circ} \mathrm{C}$ entsteht neben Silicium und Tellur grauweißes, kubisches Siliciummonotellurid SiTe.

Die Analyse ergab im Mittel 10,0\% Si (theor. 9,91\%) und $90,0 \% \mathrm{Te}$ (theor. 90,09\%). Die Einwaagen zu den Analysen und die Röntgenuntersuchungen wurden unter Luftausschluß ausgeführt. Die Dichte ergab sich zu 4,39.

$\mathrm{SiTe}_{2}$ kristallisiert im Cadmiumjodidtyp mit den Achsen $a=4,28 \pm 0,01 \AA$ und $c=6,71 \pm 0,01 \AA$. Die Intensitäten der Interferenzen auf den Drehkristall-Weissenberg- und Pulveraufnahmen ergaben einen $z$-Parameter von 0,265

1 A. W e is s u. A. W e is s, Z. Naturforschg. 7 b, 483 [1952]. $\pm 0,005$. Daraus folgt ein kürzester Si-Te-Abstand von $3,04 \AA$. Dieser Abstand ist größer als die Summe der Ionenradien nach Gold s chmidt mit $2,50 \AA$ und entspricht eher dem Abstand für metallische Bindung.

Bei der Darstellung des $\mathrm{SiTe}_{2}$ wurden gelegentlich grauweiße Fasern erhalten, welche eine faserige Modifikation des SiTe, darstellen; diese ist wahrscheinlich mit dem faserigen $\mathrm{SiSe}_{2}{ }^{1}$ isotyp.

Eine ausführliche Veröffentlichung erscheint in Kürze in der Zeitschrift für anorganische Chemie.

\section{Zur Kenntnis von Siliciumdiselenid-Glas (III. Mitt. über Siliciumchalkogenide) ${ }^{1}$}

Von Alarich Weis und Armin Weis s

Eduard-Zintl-Institut für anorganische und physikalische Chemie der Technischen Hochschule Darmstadt

(Z. Naturforschg. 8 b, 104-105 [1953]; eingeg. am 26. Januar 1953)

Glasiges Siliciumdiselenid, $\mathrm{SiSe}_{2}$, bildet sich, wenn man kristallisiertes, faseriges $\mathrm{SiSe}_{2}{ }_{2}$ im evakuierten Quarz-

1 2. Mitt.: A. W e i s s u. A. W e is s, Z. Naturforschg. 8 b, 104 [1953], voranstehend.

2 A. W e is s u. A. We is s, Z. Naturforschg. 7 b, 483 [1952]. 